\title{
La consulta previa como herramienta de la multiculturalidad en contextos migratorios: Evidencia desde Nuevo León, México ${ }^{1}$
}

\author{
Prior consultation as a tool for multiculturalism in migratory contexts: Evidence \\ from Nuevo León, Mexico
}

\author{
Claire Wright ${ }^{2}$ \\ Universidad de Monterrey, México \\ Víctor Néstor Aguirre Sotelo ${ }^{3}$ \\ Universidad Autónoma de Nuevo León, México
}

\begin{abstract}
RESUMEN En los Estados latinoamericanos, las migraciones de los indígenas del campo a la ciudad tienen ya tintes históricos, no obstante, en los últimos años dichos flujos se han acelerado, debido a conflictos extractivos en sus territorios ancestrales y la búsqueda de empleo. En este contexto, los gobiernos locales de ciudades que no cuentan con una población indígena autóctona se encuentran en una situación inesperada de diversidad étnica que demanda una
\end{abstract}

1. 1) El Proyecto de Ciencia Básica CB-2014-240556 de CONACYT (Consejo Nacional de Ciencia y Tecnología) "Procesos de Consulta Indígena en América Latina. Un análisis desde la perspectiva de la participación ciudadana"; y 2) el Proyecto SEI UIN 16514 de la Universidad de Monterrey "La Participación de los Pueblos Indígenas en México: un análisis de la implementación del derecho al Consentimiento Previo Libre e Informado".

2. Doctora en Procesos Políticos Contemporáneos de la Universidad de Salamanca. Profesora titular, Universidad de Monterrey (México), Miembro del Sistema Nacional de Investigadores (SNI) de CONACYT. Correo electrónico: cegwright@gmail.com

3. Doctor en Filosofía Acentuación en Ciencias Políticas por la Universidad Autónoma de Nuevo León (2014), Profesor de tiempo completo de la Facultad de Ciencias Políticas y Administración Pública de la misma universidad. Correo electrónico: victor.aguirrest@uanl.edu.mx 
respuesta multicultural. Así es el caso de Nuevo León, un Estado industrial en el noreste de México, que ha recibido intensos flujos de migrantes indígenas desde la década de 1990, de forma que en 2015 más de cuatrocientas mil personas ahí residentes se autoadscribían como indígenas.

A partir de trabajo de campo realizado entre 2015 y 2017, se analiza la consulta previa -que se suele asociar con conflictos territoriales- como herramienta de multiculturalidad frente a flujos de migración indígena a contextos urbanos. Se sugiere que, aun cuando las consultas realizadas no cumplan ni con los estándares del Derecho Internacional ni con los preceptos básicos de la participación ciuda- dana, pueden ofrecer un espacio simbólico que acerca a las partes para conocerse y entablar un incipiente diálogo intercultural.

PALABRAS CLAVE Pueblos indígenas, migrantes, consulta previa, multiculturalidad, México.

ABSTRACT Indigenous migration from rural to urban areas in Latin America has historical roots, however over recent years these flows have accelerated due to conflicts over the extraction of natural resources within the ancestral territories of indigenous peoples and the search for employment. In this context, local governments of areas which are not home to an autochthonous indigenous population find themselves in an unexpected situation of ethnic diversity which requires a multicultural response. Such is the case of Nuevo León, an industrial State in Mexico's North East, which has received intense flows of indigenous migrants since the 1990s, to the extent that in 2015 more than 400,000 residents self-identified as indigenous. Based on fieldwork carried out between 2015 and 2017, this article analyses prior consultation -which is usually associated with conflict in indigenous territories - as a tool for multiculturalism in the face of migratory flows of indigenous people to urban centers. The study suggests that, even when the consultations carried out do not meet the standards of international law or the basic precepts of citizen participation, they can offer a symbolic space in which to bring different actors together and work towards intercultural dialogue.

KEYWORDS Indigenous peoples, migrants, prior consultation, multiculturalism, Mexico. 


\section{Introducción}

México es un país con una gran diversidad étnica, donde vive una población indígena que, de acuerdo con los últimos datos del Instituto Nacional de Estadística y Geografía de 2015, constituye alrededor del 20\% de la población total, es decir, más de veinticinco millones de mexicanos ${ }^{4}$. Mientras que la gran mayoría de los pueblos indígenas se asientan sobre sus tierras ancestrales -en estados incluyendo Chiapas, Yucatán, Oaxaca y Veracruz - también es importante señalar un proceso de migración que empezó a acelerarse en la década de 1990, hacia centros urbanos e industriales en el norte del país, incluyendo Nuevo León ${ }^{5}$. En efecto, de acuerdo con los mismos datos de la Comisión Nacional para el Desarrollo de los Pueblos Indígenas (CDI) para 2012, Nuevo León representa el mayor aumento de población indígena en términos porcentuales experimentado por cualquier estado mexicano entre 2000 y 2010. Según la encuesta Intercensal del INEGI de 2015, más de cuatrocientas mil personas residentes en el Estado se autoadscriben como indígenas.

Las lenguas más habladas entre los indígenas migrantes asentados en Nuevo León son: el náhuatl, el huasteco, el otomí, el zapoteco y el mixteco. Los migrantes indígenas provienen principalmente de San Luis Potosí, Veracruz y el Estado de México y habitan en mayor número en los municipios del área metropolitana, incluyendo: Monterrey, Guadalupe, Escobedo, San Pedro, García y Apodaca ${ }^{6}$. Los indígenas que migran desde sus lugares de origen hacia Nuevo León lo hacen debido a la percepción de mayores oportunidades de trabajo y aprovechan las redes familiares y comunitarias que facilitan su inserción social y laboral7 . Aunque algunos grupos buscan asentarse en forma conjunta, también se produce la fragmentación de las comunidades en la ciudad, por lo que puede llegar a ser difícil reproducir la vida comunitaria que tenían en su lugar de origen ${ }^{8}$. No obstante, sería equivocado asumir que al abandonar sus comunidades los migrantes dejan de ser indígenas, dado que sus reclamos son de índole tanto social como cultural. Además, en el caso de Nuevo León se han fundado diversas organizaciones para proteger sus intereses colectivos como migrantes indígenas $^{9}$, incluyendo Enlace Potosino, Zihuakali, Zihuame Mochilla, y Procuración de Justicia Étnica, entre otras.

Sin duda alguna, la llegada de población indígena de otros estados a un estado que ya no cuenta con población indígena autóctona constituye un nuevo e importante fenómeno social y un reto para los gobiernos tanto en el ámbito municipal como estatal.

4. Dicha cifra corresponde con el indicador de la autoadscripción como indígena.

5. WRIGHT y AGUIRRE (2015). 6. DURIN (2003).

7. INEGI (2004); DURIN (2003).

8. CERDA y GALLEGOS (2008).

9. AGUIRRE y WRIGHT (2016). 
Implica la necesidad de buscar respuestas desde la multiculturalidad e, incluso, prepararse para transformaciones del propio aparato estatal en el ámbito local. Se busca analizar el reconocimiento y la implementación del derecho a la consulta previa de los pueblos indígenas en contextos de migración interna, partiendo de la premisa de que dicho derecho se ejerce con mayor dificultad cuando el sujeto se encuentra fuera de su territorio o lugar de origen, dado que este debe construir lazos nuevos, tanto con la comunidad de origen como con la población y las autoridades locales. Por lo tanto, en este contexto que se podría llamar "menos esperado", es de interés ver cómo el Estado ha ido incorporando elementos de la multiculturalidad, por procesos participativos que, en cualquier caso, distan de ser perfectos desde un punto de vista formal.

Este artículo se estructura de la siguiente manera: En primer lugar, se plantea un marco conceptual en el que se discuten conceptos clave, incluyendo la multiculturalidad y el pluralismo para señalar, luego, un importante desarrollo en el sistema internacional de derechos humanos en el caso de los pueblos indígenas: el reconocimiento del derecho a la consulta previa. Después, se propone la participación ciudadana como una perspectiva adecuada para analizar los procesos participativos realizados entre el Estado y los pueblos indígenas, incorporando aportes también de la planificación urbana. En el tercer apartado, se proporciona un breve panorama acerca de los derechos políticos de los pueblos indígenas y el derecho a la consulta previa tanto en México, en general como en el Estado de Nuevo León, en particular. En el cuarto y último apartado, se ofrece un análisis de dos consultas realizadas con migrantes indígenas en el Estado de Nuevo León, evaluándolas desde la perspectiva de la participación ciudadana. En el análisis, se emplean diversas técnicas de investigación de corte cualitativo, incluyendo la revisión de normas legales e informes de instancias gubernamentales y organizaciones de la sociedad civil (OSC), trabajo etnográfico y entrevistas informales con informantes clave. A modo de conclusión, se brindan algunas reflexiones acerca de los principales hallazgos del estudio, así como sus implicaciones para otros contextos de migración indígena en América Latina.

\section{Multiculturalidad, pluralismo y el derecho a la consulta previa de los pueblos indígenas}

Los Estados-nación, históricamente, han buscado eliminar la diversidad étnica con el afán de forjar patria y facilitar la tarea de gobierno ${ }^{10}$. Hasta hoy existe un discurso generalizado en el que las minorías deben asimilarse para poder disfrutar de los beneficios del progreso, ya que viven en un estado de "atraso", debido a su cultura ${ }^{11}$. No obstante, también existen discursos basados en la multiculturalidad que reconocen

10. DÍAZ-POLANCO (2006).

11. RUBIO y KYMLICKA (1999). 
la diversidad étnica presente en las sociedades y, además, buscan ofrecer soluciones a los retos que esta diversidad conlleva, buscando eliminar la marginación y la exclusión de las minorías culturales, sin exigir su asimilación. En vez de buscar una sociedad homogénea, la multiculturalidad busca una sociedad justa ${ }^{12}$, en la que se garantice la igualdad a la vez que se respete la diferencia. En este paradigma, es necesario garantizar el derecho a la identidad cultural, así como los derechos de las minorías étnicas ${ }^{13}$; dichos derechos serán tanto individuales (para garantizar su libertad) como colectivos (para garantizar su propia supervivencia como comunidad) ${ }^{14}$. Una vez garantizado lo anterior se busca que se establezca una interacción, un diálogo y un reconocimiento de igualdad y de respeto entre las diferentes culturas, en este momento se habla de interculturalidad, la cual se entiende como el reconocimiento de igual valor para todas las culturas en convivencia, buscando un equilibrio entre integrarse a la sociedad de acogida y el respeto a la diversidad cultural de los migrantes indígenas ${ }^{15}$. La interculturalidad, por lo tanto, representa una fase más avanzada que la multiculturalidad.

Cabe recordar que los Estados-nación se sostienen en regímenes políticos y que los regímenes democráticos se basan en la idea de la igualdad, buscando la participación de todas las personas en las decisiones públicas ${ }^{16}$. Además de la igualdad, otro pilar de la democracia sería el pluralismo, caracterizado por la tolerancia, respeto y justicia frente a la diversidad de opiniones ${ }^{17}$. Dicho lo anterior, varios autores -incluyendo Chantal Mouffe, Michael Walzer y Iris Marion Young- argumentan que la base pluralista de los regímenes democráticos resulta muy débil, debido a su pretensión de una ciudadanía igualitaria y no una ciudadanía diferenciada ${ }^{18}$. Por esta deficiencia estructural es que las poblaciones excluidas hoy exigen su participación, lo que implica la necesidad de crear nuevas instituciones que fortalezcan la deliberación pública con una perspectiva multicultural ${ }^{19}$. De acuerdo con Boaventura de Sousa Santos ${ }^{20}$, además de una educación basada en la reciprocidad cultural, una democracia pluralista tendrá diversas características de tipo institucional, que incluyen: la coexistencia de diferentes formas de deliberación democrática; diferentes criterios de representación

12. ETZIONI (2001).

13. KYMLICKA (2003).

14. CHACÓN (2005).

15. ELÓSEGUI ITXASO (2010)

16. ZORNOZA (2011) p. 132.

17. SCHMELKES (2010).

18. ZORNOZA (2011) p. 136.

19. Op. cit.

20. DE SOUSA (2010) pp. 118-119. 
democrática; el reconocimiento de derechos colectivos y el reconocimiento de nuevos derechos fundamentales de índole cultural.

Se busca trazar un incipiente proceso de construcción de una perspectiva multicultural en el ámbito local, analizando el reconocimiento de derechos políticos diferenciados y su posterior implementación en el Estado de Nuevo León. Estas páginas se enfocan en los migrantes indígenas al interior del estado mexicano y, por lo tanto, se trata de una suerte de combinación entre dos grupos minoritarios: indígenas y migrantes. Esta situación de indígena migrante -que es cada vez más común en el norte de la república mexicana- representa, entonces, una situación de potencial exclusión "de doble partida". En ese sentido, parecería ser más fácil que los grupos minoritarios logren sus demandas cuando cuentan con justicia, cultura y territorios propios ${ }^{21}$. Por lo tanto -aunque se trate del mismo Estado-nación- cuando los pueblos indígenas migran pueden tener mayores dificultades para articular sus demandas que aquellos que se asientan en sus territorios y comunidades ancestrales. La forma en la que se pueden crear espacios para el reclamo de demandas con una perspectiva de inclusión, pero con respeto a la diferencia, la migración indígena proveniente de áreas rurales hacia el área urbana de Nuevo León constituye el objetivo principal del presente artículo.

Por todo lo anterior, el reconocimiento jurídico del derecho a la participación política de los grupos minoritarios al interior de los Estados nacionales es, sin duda, un tema de gran relevancia tanto para la cohesión social como para la consolidación democrática de los regímenes políticos ${ }^{22}$. Cuando se trata de los pueblos y comunidades indígenas, dicho derecho comprende una dimensión interna (la autonomía y el autogobierno) y una externa (la participación en los asuntos del Estado al que pertenecen $)^{23}$. En este sentido, y de cara a la construcción de sociedades con mayor grado de multiculturalidad, los desarrollos relativos al derecho a la consulta previa y al consentimiento libre, previo e informado (CLPI) en el sistema internacional han resultado de gran transcendencia e impacto en América Latina.

Es importante destacar que los desarrollos se deben en gran parte a la movilización realizada por parte de las propias organizaciones indígenas. Con la formación de movimientos fuertes en la década de 1980 y con la ayuda de redes de apoyo transnacionales en la década de 1990, los pueblos indígenas de América Latina se han convertido en un importante actor político y han buscado el reconocimiento de derechos como sujeto colectivo, incidiendo tanto en el ámbito internacional como nacional ${ }^{24}$.

21. SPINNER-HAVEL (2000).

22. Así establece, por ejemplo, el Índice de Desarrollo Democrático de América Latina (IDD- LAT). Véase www.idd-lat.org. [fecha de consulta: 23 de febrero de 2017].

23. TOMASELLI (2016)

24. BRYSK (2009); KECK y SIKKINK (1998); MARTÍ I PUIG (2007); RADCLIFFE (2007). 
Sin embargo, también es importante resaltar que los movimientos indígenas han disfrutado de distintos niveles de éxito en los diferentes Estados latinoamericanos. Por una parte, se identifican casos como Ecuador, Nicaragua y Bolivia, donde unos fuertes movimientos han llegado a ocupar puestos de poder $y$, por otra parte, a otros como México, Guatemala y Perú, donde los movimientos indígenas han sido más dispersos y menos poderosos ${ }^{25}$. El impacto de las movilizaciones ha sido más uniforme y evidente es en el sistema internacional y regional de derechos humanos.

Un gran logro de cara a la protección de los derechos indígenas después de años de intensas movilizaciones fue la adopción, en 1989, del Convenio 169 sobre Pueblos Indígenas y Tribales en Países Independientes (C169). Es importante destacar que el convenio ha tenido un impacto considerable en América Latina, dado que la mayoría de los Estados de la región ya lo han ratificado (con las notables excepciones de El Salvador, Panamá y Uruguay) en un modelo boomerang ${ }^{26}$, donde los movimientos indígenas han aprovecho la presión en el ámbito internacional para reivindicar sus derechos en el ámbito nacional. El C169 tiene tres principales características que lo han convertido en una herramienta muy potente de cara a la protección de los derechos indígenas ${ }^{27}$ : la importancia que da a la relación especial entre los pueblos indígenas y sus territorios; el respeto que ofrece para sus culturas y el reconocimiento de su participación efectiva en las decisiones que les afectan.

En su artículo 6, desarrolla el derecho a la consulta previa, que implica que los gobiernos deben consultar con los pueblos indígenas frente a cualquier medida administrativa o legislativa susceptible de afectarlos. Además, se establece la obligatoriedad de buscar el consentimiento libre, previo e informado (CLPI) de los pueblos indígenas en dichos procesos para que se orienten a la búsqueda de acuerdos y que no solamente se conviertan en ejercicios formales. Sin duda alguna, esta norma se ha convertido en un punto de referencia muy importante para aquellos países que la han ratificado, dado que los tribunales nacionales se han referido a dicho texto cuando dictan sentencias en casos que involucran a los pueblos indígenas, sobre todo en lo relativo al derecho a la consulta ${ }^{28}$. Más adelante, en 2007 , se adoptó otra norma global de gran importancia en esta materia: La Declaración de las Naciones Unidas sobre los Derechos de los Pueblos Indígenas (DNUDPI). Es importante señalar que no es una norma vinculante y, en consecuencia, carece del impacto legal y simbólico del $\mathrm{C}_{169}{ }^{29}$. No obstante, ofrece mayor desarrollo e importancia al CLPI, debido a esto

25. MARTÍ I PUIG (2007); PAJUELO (2006).

26. KECK \& SIKKINK (1998).

27. WRIGHT (2014).

28. ILO (2008).

29. WRIGHT (2014). 
se ha convertido en un punto de referencia sobre este principio ${ }^{30}$. A su vez, la Corte Interamericana ha dictado una serie de sentencias sobre el derecho a la consulta y el consentimiento previo, que han servido como punto de referencia en la región, al establecer una serie de estándares sobre ambos.

Gran parte de la literatura académica que aborda el derecho a la consulta lo hace en términos jurídicos, analizando y discutiendo los estándares internacionales y regionales aplicables ${ }^{31}$. Podemos señalar, entre otros estándares, que la consulta debe ser libre (sin coacción), previa (a la decisión tomada), informada (con información adecuada y en lenguas indígenas) y con la finalidad de obtener el consentimiento de los pueblos y comunidades involucrados. Con todo, si partimos de los estándares internacionales, muy pocos casos en la práctica cumplen los criterios básicos para considerarse como "procesos de consulta previa" como tales. En efecto, existe una gran brecha entre la esfera simbólica de los estándares internacionales y regionales, por un lado, y la implementación de políticas públicas y acciones específicas dirigidas a implementar la consulta previa, por otro ${ }^{32}$. En los últimos años se han implementado miles de procesos que llevan la nomenclatura "procesos de consulta previa" a lo largo de América Latina. Entonces, cabe preguntarse si dichos procesos deben ser tachados de entrada como simulacros o si podrían tener otra función o impacto, más allá de los muy útiles, pero también muy restrictivos estándares del derecho.

\section{La participación ciudadana como perspectiva de análisis}

Consideramos que un abordaje útil para evaluar la implementación del derecho a la consulta previa, en la práctica es la perspectiva de la participación ciudadana, entendida como la participación directa de los ciudadanos en las decisiones que les afec$\tan ^{33}$. En América Latina, la construcción de una serie de mecanismos -incluyendo asambleas, plebiscitos, referéndum, la iniciativa legislativa popular, la revocación del mandato e instancias públicas de consulta y deliberación- reconocidos como características de los regímenes políticos ${ }^{34}$ que han emergido en la tercera ola de la democracia. Si bien dichos regímenes, en su gran mayoría, siguen siendo frágiles y muchos sufren de los legados de sus precursores autoritarios, es importante rescatar esta gran capacidad de innovación institucional que han tenido los Estados de la región ${ }^{35}$.

30. FERNÁNDEZ (2012).

31. Véase, por ejemplo, DOYLE (2014) y TOMASELLI (2016), entre otros.

32. STAVENHAGEN (2012).

33. ISUNZA y OLVERA (2006).

34. Op. cit., p. 96.

35. FONT (2001). 
Por su parte, en los últimos años, se han publicado varios estudios que analizan la gestión de procesos de consulta en la práctica, abordándolos con una perspectiva más política que jurídica ${ }^{36}$. Por su parte, Almut Schilling-Vacaflor ${ }^{37}$ analiza los procesos de consulta previa sobre la explotación de los hidrocarburos en Bolivia desde la perspectiva de la participación ciudadana. En su estudio se establecen dos variables dependientes que sirven para analizar qué tan "exitoso" es un proceso de consulta: el grado de participación y la calidad de la decisión tomada. La tabla a continuación expone de forma gráfica las variables y sus indicadores, que retoman debates de la participación ciudadana, así como algunos estándares básicos del derecho a la consulta.

Tabla 1: Composición de las variables dependientes.

\begin{tabular}{|l|l|}
\hline \multicolumn{1}{|c|}{ Variable } & \multicolumn{1}{|c|}{ Indicadores } \\
\hline 1. El grado de participación & $\begin{array}{l}\text { Diálogo constante entre las autoridades; } \\
\text { Involucramiento de representantes legítimos de todas las } \\
\text { comunidades afectadas } \\
\text { Consideración para las diferencias sociales, lingüísticas y } \\
\text { culturales } \\
\text { La búsqueda del consentimiento de las comunidades } \\
\text { consultadas }\end{array}$ \\
\hline 2. La calidad de la decisión tomada & $\begin{array}{l}\text { La buena fe } \\
\text { La anterioridad de la consulta a la medida tomada La } \\
\text { implementación de los acuerdos }\end{array}$ \\
\hline
\end{tabular}

Fuente: elaboración propia a partir de SCHILLING-VACAFLOR (2012).

Al considerar la variable dependiente en términos de evaluación, también puede resultar útil retomar los aportes de Sherry Arnstein ${ }^{38}$ desde la planificación urbana con su ahora famosa "escalera de participación ciudadana". De acuerdo con la autora, cualquier proceso participativo puede evaluarse y ubicarse en una escala donde los escalones de arriba corresponden al poder ciudadano (control ciudadano, delegación del poder y colaboración), los de en medio corresponden al simbolismo (apaciguamiento, consulta e información) y los de abajo a la no participación (terapia y manipulación). Al establecer categorías de esta forma, la propuesta de la autora resulta útil y se puede aplicar de forma conjunta con las variables e indicadores que nos ofrece Schilling-Vacaflor ${ }^{39}$.

36. Véase, por ejemplo, FALLETI \& RÍOFRANCOS (2018) y FLEMMER \& SCHILLING-VACAFLOR (2015), entre otros.

37. SCHILLING-VACAFLOR (2012).

38. ARNSTEIN (1969).

39. SCHILLING-VACAFLOR (2012). 
Por otro lado, en términos de los factores que pueden influir en el éxito de los procesos de consulta, en el mismo estudio de Almut Schilling-Vacaflor se menciona una serie de variables e indicadores que corresponden con tres dimensiones señaladas en las discusiones sobre la participación ciudadana, es decir, el diseño institucional, factores contextuales-relacionales y factores comunicacionales-cognitivos. La tabla a continuación recoge las tres variables independientes y sus respectivos indicadores.

Tabla 2. Composición de las Variables Independientes

\begin{tabular}{|l|l|}
\hline \multicolumn{1}{|c|}{ Variable } & \multicolumn{1}{c|}{ Indicadores } \\
\hline 1. El diseño institucional & $\begin{array}{l}\text { Un marco legal progresivo y específico. La natu- } \\
\text { raleza vinculante de los acuerdos. }\end{array}$ \\
\hline 2. Factores contextuales-relacionales & $\begin{array}{l}\text { Comunidades con influencia y poder de movili- } \\
\text { zación. } \\
\text { El apoyo de organizaciones internacionales de dere- } \\
\text { chos humanos. } \\
\text { Pocas asimetrías de poder y desigualdades. }\end{array}$ \\
\hline 3. Factores comunicacionales-cognitivos & $\begin{array}{l}\text { Comunidades con conocimientos e información. } \\
\text { La realización de debates públicos y escrutinio por } \\
\text { los medios de comunicación masivos. }\end{array}$ \\
\hline
\end{tabular}

Fuente: elaboración propia a partir de SCHILLING-VACAFLOR (2012).

Es importante recordar que el presente estudio es de corte cualitativo y, por lo tanto -más que encontrar pautas explicativas a través de relaciones estadísticas significativas-, las variables e indicadores planteados atrás ofrecerán una guía de categorías para el análisis de los dos procesos de consulta abordados en estas páginas.

\section{Los pueblos indígenas y sus derechos políticos en México y Nuevo León: una contextualización}

El primer paso formal hacia la multiculturalidad en el ámbito federal se dio con la reforma a la Constitución Política de los Estados Unidos Mexicanos (CPEUM) en 1992, cuando se reconoció por primera vez la composición pluricultural del Estado. Más adelante, en 2001 -ya varios años después del levantamiento zapatista- el artículo 2 de la CPEUM se reformó, profundizando en la multiculturalidad y el pluralismo del Estado. En estas reformas se reconocieron la existencia de los pueblos y comunidades indígenas, la importancia de su autoadscripción en tal de indígenas y la aplicación de sus sistemas normativos. De la misma forma, se garantizaron sus derechos colectivos, incluyendo la libre determinación y autonomía, así como la obligación de la federación, los estados y los municipios para asegurar su participación a la hora de proteger sus derechos y promover su pleno desarrollo. La CPEUM también ofrece protección 
para sus tierras y territorios y contempla medidas en contra de la discriminación. Si bien la reforma es de cierta transcendencia, también es importante señalar que es mucho más limitada de lo que se pactó en los Acuerdos de San Andrés con los zapatistas y que, más allá del reconocimiento formal de sus derechos, el disfrute de los mismos por parte de los pueblos indígenas sigue siendo muy limitado, debido, entre otros factores, a la falta de políticas públicas focalizadas hacia ellos. En este sentido, México -igual que en gran parte de América Latina- sufre de una considerable "brecha de implementación", es decir, hacer efectivos los derechos plasmados en el ámbito formal ${ }^{40}$.

Respecto al derecho a la consulta y al CLPI, en el ámbito federal, hasta la fecha no se ha aprobado ley alguna sobre esta materia. Es importante destacar que esta situación tampoco es única en la región latinoamericana, dado que solo dos países, Perú y Panamá, han aprobado una ley de consulta indígena ${ }^{41}$. En cualquier caso, es importante destacar que en 2011 se llevó a cabo un proceso de consulta sobre el Anteproyecto de Ley General de Consulta a Pueblos y Comunidades Indígenas, pero no prosperó y México sigue sin una ley de consulta en el ámbito federal. Por su parte, la CDI ha elaborado un protocolo para la implementación de consulta a los pueblos indígenas tomando como referencia estándares del C169, situación que refleja la importancia de los desarrollos normativos internacionales en políticas domésticas ${ }^{42}$.

Resulta difícil identificar con precisión cuántos procesos de consulta se han realizado en México por tres principales razones: 1) no existe un organismo especializado que se encargue de los procesos de consulta, si bien la CDI cuenta con una dirección de participación y consulta previa en el ámbito federal, pero solo le corresponde ofrecer tanto apoyo técnico como financiero al organismo que tiene la competencia sobre la temática objeto de la consulta ${ }^{43}$; 2) existen procesos de "consulta" que no quedan registrados en informes oficiales y 3) en muchos casos, lo que se le llama "consulta previa” es, más bien, un ejercicio de sondeo básico, algunas veces para obtener información de los pueblos indígenas. Por su parte, la CDI ha producido informes sobre trece procesos de consulta previa en el ámbito federal, sobre todo en materias relacionadas con la formulación de políticas públicas. Otros procesos de consulta se han realizado por parte de diversas autoridades federales, incluyendo Secretaría de Energía (SENER), la Comisión Intersecretarial de Bioseguridad de los Organismos Gené-

40. MARTÍ I PUIG (2007).

41. WRIGHT (2014).

42. El protocolo se puede consultar en el siguiente enlace: www.gob.mx/cms/uploads/ attachment/ file/37009/cdi_protocolo_consulta_pueblos_indigenas_2014.pdf [fecha de consulta: 23 de marzo de 2016].

43. Información de una entrevista informal llevada a cabo con representante de la CDI por parte de los autores del capítulo. 
ticamente Modificados (CIBIOGEM), Comisión Nacional del Agua (CONAGUA), el Instituto Nacional de Evaluación Educativa (INEE) y el Instituto Nacional Electoral (INE), entre otras. Parece que muchas autoridades estatales (y algunas municipales) también han realizado procesos de consulta en los últimos años, pero no existe un registro definitivo de estas experiencias.

Si bien el reconocimiento e implementación del derecho a la consulta previa de los pueblos y comunidades indígenas en México quedan muy rezagados en comparación con Estados como Colombia o Perú, es importante señalar que existe una creciente conciencia entre las autoridades de diferentes niveles de gobierno sobre la necesidad de realizar procesos de consulta antes de tomar decisiones administrativas o legislativas que podrían afectar a la población indígena. En gran parte esta conciencia tiene que ver con procesos de litigio realizados por organizaciones indígenas y sentencias de diversos tribunales (incluyendo la Suprema Corte de Justicia de la Nación (SCJN) y el Tribunal Electoral del Poder Judicial de la Federación (TEPJF), entre otros) que reconocen el derecho a la consulta y obligan a las autoridades a tomar medidas para garantizarlo. Es importante destacar que este proceso de impulso a la consulta previa a través del Poder Judicial se da en el contexto de la reforma de 2011 en materia de derechos humanos, cuando la SCJN determinó que los tratados internacionales tenían el mismo rango jurídico que la Constitución. A partir de ahí, el alcance del derecho a la consulta previa en México cobró una renovada importancia, tanto en procesos nacionales como en procesos locales.

Dada la situación anterior, y teniendo en cuenta la composición federal de la república mexicana, también es de gran interés voltear la mirada en el ámbito local dado que en muchos casos ahí es donde se da el principal contacto entre la población indígena y las autoridades del Estado. Además, cabe señalar que, en el marco de la reforma a la CPEUM de 2001, se delegó la facultad de legislar sobre los derechos indígenas a los Estados. En este sentido, se observa que existen situaciones muy diversas respecto al reconocimiento de los derechos indígenas, incluyendo el derecho a la consulta previa, en el ámbito local en México. Por ejemplo, mientras que San Luis Potosí y Durango cuentan con sus respectivas leyes de consulta, otros Estados incluyendo Aguascalientes, Coahuila, Tamaulipas y Zacatecas no cuentan ni siquiera con una ley de derechos indígenas. En contextos migratorios -como es el caso de Nuevo León- la situación cobra características muy específicas dado que el vínculo ancestral con el territorio - tal y como se plantea en el C169 y la DNUDPI- sufre cambios importantes. A continuación, se ofrecen algunas experiencias recientes del Estado de Nuevo León con el objetivo de arrojar luz sobre los desafíos enfrentados por gobiernos locales a la hora de asumir una situación de diversidad étnica provocada por flujos migratorios repentinos y acelerados. 
Como se planteó en la introducción, existe un importante fenómeno de migración indígena hacia el noreste de México. Ya en 2003 en su informe sobre México, el relator especial de las Naciones Unidas Rodolfo Stavenhagen resaltó la marginación social de los pueblos indígenas en toda la república mexicana y la carencia de una política pública específica para los migrantes indígenas a zonas urbanas. En el caso neoleonés, la respuesta a la migración indígena ha pasado por varias estrategias realizadas por el gobierno federal y el gobierno estatal, incluyendo un programa de educación bilingüe intercultural, una serie de centros comunitarios interculturales y la apertura de una oficina de extensión de la CDI en Nuevo León $y^{44}$. En el caso de los municipios, en el municipio de Monterrey se ha formado un órgano encargado de asuntos indígenas que -en cualquier caso- no cuenta con representantes indígenas entre sus miembros y en 2018 se abrió una oficina de atención a la población indígena y migrante en el municipio de San Pedro Garza García. Más allá de estos dos ejemplos, no se han detectado otras estrategias específicas por parte de los doce municipios que conforman la Zona Metropolitana de Monterrey, si bien se aprecia la presencia de funcionarios en talleres de sensibilización realizados por organismos como la Comisión Estatal de Derechos Humanos y en actividades y eventos puntuales para marcar fechas importantes, incluyendo el Día Internacional de los Pueblos Indígenas.

Por parte de los migrantes indígenas, el proceso de migración dificulta, pero no rompe los lazos con su comunidad de origen e, incluso, en algunos casos se reconstruyen dinámicas comunitarias en la ciudad. En cualquier caso, dada la diversidad étnica en su interior, se ha dado lugar a una serie de organizaciones panétnicas que buscan representar los intereses de los indígenas en la ciudad, incluyendo: Enlace Potosino, Zihuakali, Zihuame Mochilla, y Procuración de Justicia Étnica. Además de su labor comunitaria, donde más se ha sentido la presión de dichas organizaciones en el Estado, es la exitosa demanda del reconocimiento jurídico, siguiendo un patrón que -como se señaló anteriormente- se ha producido en el ámbito internacional y en toda América Latina. Los migrantes indígenas, las organizaciones sociales que buscan defender sus intereses y los actores gubernamentales favorables hacia su cau$\mathrm{sa}^{45}$ lucharon por su reconocimiento jurídico, ya que salvo en el ámbito educativo, la constitución y las leyes de Nuevo León hacían escasa referencia al reconocimiento a la población indígena en la entidad. Es importante recordar además que, a partir de la reforma constitucional en materia indígena en el ámbito federal en 2001, correspondía a los Estados realizar los cambios necesarios en sus marcos normativos.

44. WRIGHT \& AGUIRRE (2015).

45. Entrevista personal con representante de la Procuración de Justicia Étnica llevada a cabo por los autores en Monterrey, 19 de mayo de 2015. 
La demanda por el reconocimiento por parte de los migrantes indígenas al Estado de Nuevo León se cumplió en febrero del año 2012, cuando se llevó a cabo la reforma al artículo 2 de la Constitución del Estado, que reconoce que "El Estado de Nuevo León, tiene una composición pluriétnica, pluricultural, multilingüística, a la que contribuyen los indígenas asentados en su territorio". Además, no es tan amplio como el artículo 2 de la Constitución Federal, sin embargo, reconoce los derechos sociales, políticos y culturales de este sector. En cuanto a los derechos culturales y de identidad se establece que los indígenas en la Entidad:

tienen derecho a preservar y enriquecer sus lenguas y conocimientos; colaborar en la protección de su hábitat, patrimonio cultural, lugares de culto y demás elementos que constituyan su cultura e identidad; a decidir sobre sus normas internas de convivencia, organización social, económica, política y cultural.

Constitución del Estado de Nuevo León, artículo 2

El respeto hacia las normas internas de organización política de los pueblos indígenas representa un importante reconocimiento de sus derechos políticos en una dimensión interna y un primer paso en la construcción de una democracia multicultural en el ámbito local. Es más, el mismo artículo 2 también reconoce el derecho a la consulta: "Se consultará a los indígenas, e incorporarán sus recomendaciones, para la elaboración de los Planes Estatal y municipales de desarrollo", si bien no ofrece mayores detalles al respecto. En este sentido es un espejo del artículo 2 de la CPEUM y, por lo tanto, se queda muy corto de los estándares internacionales sobre la materia.

Asimismo, en junio de 2012 se publicó la Ley de Derechos Indígenas en el Estado de Nuevo León, que tiene como objetivo reglamentar el artículo 2 de la Constitución local y va un paso más allá en sus contenidos. Además de reconocer derechos sociales, incluyendo el derecho a la educación y el derecho a la salud, se reconocen los sistemas normativos indígenas y, de la misma forma, se les reconoce la autonomía para que se puedan organizar libremente y preservar sus prácticas sociales, económicas, políticas y culturales. También se establecen dos tipos de derechos políticos de suma importancia: el derecho a la representación y el derecho a la participación.

Por un lado, se determina que "los indígenas tienen el derecho de elegir a quien los represente ante el Ayuntamiento respectivo” asentando, además, en el artículo 9:

Los ayuntamientos de los Municipios en los que estén asentados los indígenas, deberán crear órganos o comisiones encargados de atender sus asuntos; sus titulares o integrantes deberán respetar en su actuación los usos y costumbres de los indígenas.

Ley de Derechos Indígenas del Estado de Nuevo León, artículo 9 
Por otro lado, también se incluye el derecho a la consulta previa y, si bien se circunscribe a los planes estatal y municipales de desarrollo, se incluyen varios estándares internacionales sobre la materia:

El Estado y los Municipios podrán consultar a los indígenas en la elaboración del Plan Estatal y Planes Municipales de Desarrollo, mediante procedimientos apropiados, de buena fe y en particular a través de organizaciones representativas, y en lo procedente y viable a incorporar las propuestas que realicen.

Ley de Derechos Indígenas del Estado de Nuevo León, artículo 28

Aunque resulta muy limitado en su alcance, la inclusión del derecho a la consulta tanto en la Constitución como en la Ley de Derechos Indígenas refleja la incorporación en el ámbito local de avances normativos que se están produciendo en toda América Latina y de manera paulatina en México en el ámbito federal ${ }^{46}$. También refleja pasos tentativos en la construcción de una democracia multicultural en un nuevo contexto de diversidad étnica, que es producto de recientes olas migratorias desde otros Estados mexicanos. En ese sentido, es importante señalar que no todos los Estados mexicanos han reconocido el derecho a la consulta en sus marcos normativos, con las ausencias más notables entre aquellos Estados que carecen de poblaciones autóctonas entre sus habitantes. De forma clave, las reformas legales producidas en Nuevo León fueron precedidas por una consulta con diversas organizaciones indígenas $^{47}$, reconociendo la importancia de involucrar a los mismos indígenas en las decisiones que se toman en su nombre, una experiencia que se detallará en el siguiente apartado.

Cabe destacar la importancia simbólica de la aprobación de la reforma constitucional y la Ley de los Derechos Indígenas en Nuevo León en el año 2012. Fue a partir de este año que proliferaron las OSC relacionadas con la problemática indígena en el Estado y en 2013 se produjeron protestas para exigir su implementación ${ }^{48}$. La importancia del reconocimiento jurídico para los propios indígenas en contextos migratorios no debe subestimarse, aunque, como ocurre en toda América Latina, la dificultad reside en la llamada "brecha de implementación". Hasta la fecha, por ejemplo, no se han producido procesos de consulta previa sobre los planes municipales de

46. Sobre este tema y su importancia para los pueblos indígenas de América Latina, véase WRIGHT (2014).

47. Entrevista personal con representante de la Procuración de Justicia Étnica llevada a cabo por los autores en Monterrey, 19 de mayo de 2015.

48. AGUIRRE y WRIGHT (2016). 
desarrollo ${ }^{49}$. No obstante, autoridades de gobierno federal y estatal han organizado dos procesos participativos relevantes que merecen detallarse y analizarse con cierta profundidad.

\section{Análisis de procesos participativos con los pueblos indígenas en Nuevo León desde la perspectiva de la participación ciudadana}

A continuación, se describen y analizan dos procesos participativos que se llevaron a cabo con migrantes indígenas en Nuevo León usando la nomenclatura de "consulta". Se hará hincapié en la medida en la que la experiencia se puede considerar exitosa -desde la perspectiva de la participación ciudadana-y las razones que influyeron en ello en cada caso. El análisis llevado a cabo y que se presenta en esta sección se basa en la revisión de documentos, veinte entrevistas informales con informantes clave, como representantes indígenas, dirigentes de OSC, funcionarios gubernamentales relacionados con el proceso de la consulta previa y académicos. Se decidió por las entrevistas informales debido a que ambos procesos se desarrollaron en condiciones diferentes, como se explicará más adelante, por lo que las entrevistas que se planearon fueron flexibles, y se adaptaron al papel que el entrevistado desempeñaba en relación con la consulta. Además, se llevó a cabo trabajo etnográfico, asistiendo como parte del público a la Consulta Nuevo León en el parque La Alameda de Monterrey, todo lo anterior realizado en Nuevo León entre 2013 y 2018.

\subsection{La consulta previa sobre la Ley de Derechos Indígenas (2011)}

En el apartado anterior se detalló el reconocimiento jurídico de los derechos políticos de la población indígena asentada en Nuevo León, que se dio tanto en la reforma a la Constitución estatal (en su artículo 2) como en la Ley de Derechos Indígenas. Es importante destacar que en 2011 -el año anterior a las reformas legales referidas- se produjo un proceso de consulta por la Secretaría de Desarrollo Social de Nuevo León con el apoyo técnico de la CDI y la asesoría de instituciones de educación superior de la entidad. De acuerdo con el proyecto de consulta ${ }^{50}$, el proceso tuvo como objetivo

49. Esto no significa que las autoridades no tengan sensibilidad hacia la diversidad étnica en sus municipios. En enero 2016 se celebró una consulta sobre el Plan Municipal de San Pedro Garza García y se incluyó la temática de grupos vulnerables/minorías étnicas, invitando a expertos en la materia y representantes de organizaciones indígenas. Lejos de ser una consulta previa con comunidades indígenas, sí demuestra una preocupación por incluir la temática y los representantes más visibles en la planificación municipal.

50. Proceso de Consulta para la Elaboración del Proyecto de Iniciativa de Reforma Constitucional y Ley Reglamentaria en Materia de Derechos y Cultura Indígena en el Estado de Nuevo León. Documentado facilitado a los autores por la CDI en Nuevo León. 
general "generar un marco jurídico local favorable para hacer vigentes los derechos y la cultura de la población indígena en la entidad y crear mejores condiciones de bienestar para las personas y comunidades indígenas" y, a la vez, estableció cuatro objetivos específicos:

1. Consultar a los principales actores que tienen relación con el reconocimiento de los derechos y la cultura de los pueblos indígenas en la entidad.

2. Promover la participación informada y activa de los sujetos indígenas.

3. Contar con un documento que refleje las opiniones, propuestas y aportaciones de los actores consultados, que sirva de insumo a las instancias correspondientes para mejorar el diseño de un proyecto de iniciativa de reforma constitucional y ley reglamentaria en materia de derechos y cultura indígena.

4. Contribuir a la creación de un marco jurídico eficaz y eficiente en su aplicabilidad, que redunde en beneficio de la población indígena en el Estado.

En el proceso de planificación se dio mucha importancia a definir la población sujeta a consulta, sobre todo dada la dificultad a la hora de reconocer las autoridades tradicionales en contextos migratorios. Por esta razón, se optó por llevar a cabo consultas con los siguientes grupos: asambleas generales de los asentamientos y organizaciones indígenas, representantes de asentamientos indígenas, representantes de organizaciones indígenas, estudiantes indígenas, profesionistas indígenas y la población indígena dispersa y aislada. Además, se invitó a académicos, investigadores y funcionarios a participar en el proceso. Las consultas con los distintos grupos se llevarían a cabo en diferentes sesiones y tendrían dinámicas diferentes en cada caso. Por ejemplo, en las consultas con las organizaciones indígenas primero se ofreció una contextualización y luego se recogerían las sugerencias mientras que la consulta con la población dispersa se llevó a cabo a través de entrevistas individuales.

En cuanto al cronograma, se estableció un periodo de diez meses para la organización de la consulta (etapa previa), un mes para la convocatoria (cerrada para organizaciones o grupos fácilmente identificables y abierta para la población dispersa), un mes para la consulta en sí (etapa de consulta) y un mes para la etapa de sistematización y entrega de resultados, incluyendo un foro de consulta final sobre la propuesta para la reforma legal.

El caso de la consulta previa a la Ley de Derechos Indígenas de 2011 revela la importancia de una planificación estratégica, con suficiente tiempo, una convocatoria amplia y una buena comunicación entre actores. De acuerdo con la perspectiva de la participación ciudadana, se puede decir que el proceso fue exitoso en el sentido que fomentó la participación de los actores interesados. No obstante, en términos de la calidad de la decisión tomada, aunque la consulta fue anterior a la reforma legal, no se dio retroalimentación acerca de cómo se integraron las propuestas al proyecto de 
reforma y debido a lo anterior, es muy difícil establecer en qué medida los acuerdos se implementaron. De ahí que, de acuerdo con la escalera de participación ciudadana, se puede ubicar el caso en los escalones del "simbolismo", entre consulta e información.

En términos de los factores que influyeron en el proceso, podemos señalar que, en el plano contextual-relacional, participó una variedad de actores y, por lo tanto, el proceso fue pluralista. En el plano comunicacional-cognitivo, se dieron debates públicos y con escrutinio considerable sobre el proceso. A pesar de que en 2011 no existía un diseño institucional favorable (no se reconocieron los derechos indígenas en el Estado), no parece haber constituido una barrera para la planificación del proceso, que se fundó en principios del derecho internacional (incluyendo el C169), la legislación vigente en el ámbito federal y un ambiente generalizado de confianza mutua entre actores.

\subsection{Consulta sobre el Plan Estatal de Desarrollo (2016)}

Después de la aprobación de la reforma constitucional y de la Ley de Derechos Indígenas en el ámbito estatal, se dieron muy pocos avances en cuanto a la implementación de la nueva normatividad en el Estado. No obstante, con el cambio de gobierno en el ámbito estatal en 2015, se dio un nuevo impulso a la situación de los pueblos indígenas en el Estado, de forma que "el tema indígena" volvió a la agenda pública durante unos meses. En marzo 2016 se realizó un seminario de dos días para ofrecer un balance acerca de la implementación de esta normatividad y los avances pendientes. Unos días después del seminario, en el marco de una serie de consultas sobre varias temáticas del Plan Estatal de Desarrollo 2016-2021, se llevó a cabo un foro en el parque de La Alameda, lugar de encuentro de los migrantes indígenas en Monterrey ${ }^{51}$.

Es importante destacar que, aunque la consulta fue dirigida a la población indígena de Nuevo León la nomenclatura del evento fue "Consulta Nuevo León". El foro tomó lugar un domingo por la tarde en el teatro hundido del parque, después de una convocatoria abierta por redes sociales y panfletos en comunidades ya identificadas en la que se les invitaba a los migrantes indígenas a acercarse al foro y ver de qué manera la Secretaría de Desarrollo Social les podría ayudar. En términos del proceso en sí se combinaron dos dinámicas: 1) la aplicación de un cuestionario en que los entrevistados ordenaban sus necesidades en materia social (previamente identificadas por la Secretaría de Desarrollo Social de Nuevo León) y 2) un foro deliberativo. En esta segunda dinámica no se establecieron preguntas concretas ni se ofreció información sobre el plan de desarrollo, más bien se dejó un espacio abierto para que los participantes pudieran exponer sus necesidades. El foro duró unas tres horas en total.

51. Sobre este espacio véase DÍAZ MELÉNDEZ (2009). 
Al ofrecer un balance del proceso de consulta, es importante destacar que el grado de participación era bajo, dado que no hubo presencia significativa de autoridades de comunidades o representantes de organizaciones indígenas y, más bien, participaron algunos migrantes indígenas y otras personas que se encontraron en ese momento en La Alameda y que se acercaron por curiosidad. Además, es muy difícil establecer la calidad de la decisión tomada, ya que no quedó claro cómo se iba a tomar en cuenta los aportes de los participantes en el Plan Estatal de Desarrollo. Pensando en las razones de esta situación, es importante destacar el plano comunicacional-cognitivo y la falta de convocatoria pronta, amplia y clara sobre el evento. En este sentido, para los participantes no quedaba claro que era un evento de consulta a la población indígena y no a la población en general dado que varias personas no indígenas participaron con demandas sobre la situación de inseguridad en general. Este hecho creó un grado de confusión entre los participantes que se fue despejando a lo largo del evento. En el plano de los factores contextuales-relacionales, también es importante resaltar que al ser una participación escasa y dispersa existía cierta asimetría de poder entre autoridades y migrantes indígenas en el evento. Finalmente -aunque para esa fecha Nuevo León ya contemplaba el derecho a la consulta en su normatividad estatal- el procedimiento a seguirse en el proceso no era claro.

Por todas las razones anteriores, el caso de la consulta a la población indígena de Nuevo León sobre el Plan Estatal de Desarrollo difícilmente se puede considerar como un proceso de consulta como tal, dado que fue un evento puntual con poco alcance entre la población indígena. Sin embargo, sí se podría considerar como un evento simbólico y público que buscó tender puentes entre el gobierno del Estado y la población de migrantes indígenas. A pesar de un inicio muy tentativo con pocos comentarios y participación, durante el foro cada vez más participantes indígenas hicieron escuchar su voz para hacer algún reclamo o exponer un problema que habían tenido. Estas demandas se culminaron en varias intervenciones sobre la discriminación sufrida en el Estado, sobre todo por hablar en su propio idioma. Al ver que una mujer lloraba al relatar su decisión de no enseñar su lengua materna a su hijo, se acercó el representante de gobierno y le pidió perdón a nombre de la sociedad neoleonesa. Fue un momento de gran importancia para los asistentes en el foro, que produjo cierto cambio en el ambiente y constituyó una clara señal de buena voluntad. Por lo tanto, a pesar de sus deficiencias, es importante rescatar la importancia de esta consulta cómo encuentro entre el gobierno del Estado y la población migrante indígena en el plano simbólico. En ese sentido, si bien se puede ubicar en los escalones de "no participación" de la escalera de participación ciudadana, sería en el ámbito de "terapia" (que consiste en escuchar las demandas del ciudadano, permitir que se desahogue, pero hasta ahí llega la acción del gobierno) que en sí constituye un paso hacia la construcción de una visión más multicultural por parte de las autoridades locales. 


\section{Conclusiones}

Se ha buscado describir y analizar la paulatina construcción de una respuesta multicultural por parte de autoridades gubernamentales locales frente a importantes flujos migratorios de indígenas desde sus territorios ancestrales a centros urbanos. Concretamente, se ha detallado el reconocimiento de los derechos políticos de la población indígena migrante asentada en el Estado de Nuevo León en México, haciendo hincapié en el reconocimiento del derecho a la consulta previa como derecho clave que nace desde el sistema internacional de derechos humanos. En este caso "menos esperado", en el que los indígenas se encuentran lejos de sus tierras de origen y en que las autoridades locales cuentan con poca experiencia de atención a la diversidad étnica (debido en gran parte a la ausencia de población indígena autóctona en la entidad), se han producido avances considerables en el ámbito legal, con la reforma a la Constitución del Estado y la promulgación de una ley de derechos indígenas, en 2012. Si bien el derecho a la consulta previa se encuentra muy limitado en ambas normas (y no se da ninguna referencia al consentimiento libre, previo e informado), el hecho de que se reconozca en la legislación es un paso importante hacia la construcción de una perspectiva multicultural y pluralista en el ámbito local.

Específicamente se describen y analizan dos procesos participativos realizados con la población indígena asentada en Nuevo León bajo la nomenclatura "consulta”. Los procesos poco tienen que ver con los estándares internacionales y regionales plasmados en el C169, la DNUDPI y la jurisprudencia de la CIDH. En cualquier caso, si los abordamos desde la perspectiva de la participación ciudadana nos da un abanico más amplio y realista para evaluar estos procesos. Por una parte, la consulta sobre la Ley de Derechos Indígenas que se produjo en 2011 era amplia, abierta y llevaba una planificación muy meticulosa, si bien a la hora de incorporar los aportes de los participantes en la ley la retroalimentación por parte de las autoridades era muy escasa. Por otra parte, la consulta sobre el Plan Estatal de Desarrollo de 2016 resultó mucho más limitada en su organización y alcance, y contó con una participación muy escasa por parte de la población indígena residente en el Estado. En ese caso, lo rescatable es un avance simbólico en términos del acercamiento entre partes, el tratamiento público de temas como la discriminación (incluyendo una disculpa desde las autoridades gubernamentales) y el esfuerzo por cumplir con las disposiciones legales de cara a la protección de los derechos indígenas. En ambos casos queda patente la importancia de las variables institucional, comunicacional-organizativa, y contextual-relacional en los resultados producidos.

Sería ilusorio -e impreciso- presentar el caso de Nuevo León como un éxito en términos de la construcción de una perspectiva multicultural y plural en el Estado. Si bien se identifican avances en términos de estrategias concretas (en materia de desarrollo social y educación), reconocimiento legal y procesos participativos, tam- 
bién se han producido una serie de estancamientos. Tanto los centros comunitarios interculturales como la educación intercultural bilingüe son de un alcance limitado; la implementación de lo estipulado en la reforma constitucional y la Ley de Derechos Indígenas queda en gran parte pendiente; y los procesos participativos siguen siendo escasos y de baja calidad en términos de la efectiva participación de la población indígena. En este contexto, es importante considerar el papel de diferentes actores (los municipios, por ejemplo, no han asumido un papel claro) así como los vaivenes en las relaciones entre autoridades federales y estatales así como las OSC, dado que se han producido ciertos momentos de acercamiento y colaboración y otros momentos de alejamiento e, incluso, desconfianza. El impulso, la coordinación y la buena voluntad de todos los actores relevantes es imprescindible para ofrecer una respuesta satisfactoria hacia el desafío planteado, desde una perspectiva multicultural y pluralista.

Por lo anterior, se concluye que es difícil que los gobiernos locales creen espacios deliberativos desde la ciudadanía diferenciada, pero también se han rescatado varios avances, sobre todo en el plano simbólico. La consulta previa es tanto un derecho de los pueblos indígenas como una obligación de las autoridades gubernamentales y, aterrizado a través de procesos administrativos, puede llegar a ser una herramienta muy potente para la construcción de la multiculturalidad en contextos migratorios al acercar a las partes y obligarlas a buscar formas de encuentro y entendimiento. Es importante identificar y analizar los procesos y cambios que se dan en el ámbito local, ya que muchas veces es donde los problemas se tratan de forma directa y donde el ciudadano tiene mayor contacto con la política. Esta situación cobra gran importancia en contextos como el mexicano, debido a su composición federal, pero los hallazgos de este estudio también deben ser de interés de todas aquellas ciudades latinoamericanas que viven procesos de migración interna parecidos, como es el caso de Santiago de Chile, Quito y La Paz, entre otras. Sin duda alguna, si bien la diversidad étnica en América Latina es una cuestión histórica, la problemática sigue en constante evolución y es importante que los gobiernos respondan a los desafíos nuevos que representa. Contar con las herramientas, experiencias, prácticas y actitudes adecuadas será de gran importancia en el camino. 


\section{Referencias bibliográficas}

AGUIRRE, Víctor Néstor y WRIGHT, Claire (2016): “Organización Política y Reconocimiento Jurídico de la Población Indígena en Nuevo León”. En WRIGHT, Claire y CuevAs, Verónica Ascención (coords.), Participación ciudadana y democracia en el Estado de Nuevo León (México DF, Fontamara).

ARNSTEIN, Sherry (1969): “A Ladder of Citizen Participation”. En Journal of the American Planning Association, Scottish Government's Better Community Engagement Programme, vol. 35, No 4, pp. 216-224.

BRYSK, Alison (2009): "De la tribu a la aldea global. Derechos de los pueblos indígenas, redes transnacionales y relaciones internacionales en América Latina”. En Revista Española de Investigaciones Sociológicas, № 131, Universidad de California Santa Barbara, pp. 133-144

CERDA, José y GALLEGOS, Luz (2008): La migración indígena hacia el Noreste de México: Oportunidades y retos de la acción Institucional en el caso del Estado de Nuevo León, (Monterrey, Comisión Nacional para el Desarrollo de los Pueblos Indígenas)

CHACÓN, Oswaldo (2005): Teoría de los derechos de los pueblos indígenas. Problemas y límites de los paradigmas políticos (México DF, Instituto de Investigaciones Jurídicas-UNAM).

DE SOUSA, Boantura (2010): Refundación del Estado en América Latina. Pespectivas desde una epistemología del Sur (México, Editorial Siglo XXI, Siglo del Hombre Editores, Universidad de los Andes).

DÍAZ MELÉNDEZ, Adela (2009): Migración indígena y apropiación del espacio público en Monterrey. El caso de la Alameda (Monterrey, Centro de Estudios Históricos de la Universidad de Monterrey, Centro de Investigaciones y Estudios Superiores en Antropología Social, Facultad de Filosofía y Letras de la Universidad Autónoma de Nuevo León).

DÍAZ-POLANCO, Héctor (2006): Elogio a la diversidad. Globalizacion, multiculturalismo y etnofagia (México, Siglo XXI).

DOYLE, Cathal (2014): Indigenous Peoples, Title to Territory, Rights and Resources: The Transformative Role of Free Prior and Informed Consent (London, Routledge).

DURIN, Séverine (2003): "Nuevo León, un nuevo destino de la migración indígena". En Revista de Antropología Experimental, No 3 , Centro de Investigaciones y Estudios Superiores en Antropología Social, pp. 1-7

DURIN, Séverine (2011): Etnicidades urbanas en las Americas: Proceso de inserción, discriminación y politicas multiculturalistas (Monterrey, Centro de Investigaciones y Estudios Superiores en Antropología Social). 
ELÓSEGUI ITXASO, María (2010): "El modelo de interculturalidad en el informe de la Comisión Bouchard-Taylor para Québec”. En Anuario de Derechos Humanos. Nueva Época, No 11, Universidad Complutense, pp. 129-163.

ETZIONI, Amitai (2001): La Tercera Via hacia buena sociedad: Propuestas desde el comunitarismo (Madrid, Editorial Trotta).

FALLETI, Tulia G. \& RIOFRANCOS, Thea N. (2018): "Endogenous Participation: Strengthening Prior Consultation in Extractive Economies". En World Politics, vol. 70, No 1, Princeton University, pp. 86-121.

FERNÁNDEZ, Beatriz (2012): "Los pueblos indígenas y el sistema de Naciones Unidas: perspectivas de cambio”. En GÓMEZ, Felipe (ed.) y BERRAONDO, Mikel (ed.), Los derechos indigenas tras la Declaración (Bilbao, Universidad de Deusto), pp. 65-89.

FONT, Joan (coord.)(2001) Ciudadanos y decisiones públicas (Barcelona, Ariel Ciencia Política).

ILO (2008): Application of Convention no. 169 by domestic and international courts in Latin America. A casebook. (Geneva, International Labour Organization).

INEGI (2004): La población indígena en México (México, INEGI).

ISUNZA, Ernesto (coord.) y OLVERA, Alberto (coord.) (2006): “Introducción”. En Democracia, rendición de cuentas y sociedad civil: participación ciudadana y control social (México DF, Centro de Investigaciones y Estudios Superiores en Antropología Social, Universidad Veracruzana, Miguel Ángel Porrúa).

KECK, Margaret \& SIKKINK, Kathryn (1998): Activists beyond borders: Advocacy networks in international politics (London, Cornell University Press).

KYMLICKA, Will y RUBIO, Ruth (1999): "Liberalism and Minority Rights. An Interview". En Ratio Juris, vol. 12, No 2, Queen's University and University of Seville, pp. 133-152.

KYMLICKA, Will (2003): La politica vemacula. Nacionalismo, multiculturalismo y ciudadanía (España, Paidós Estado y Sociedad).

MARTÍ I PUIG, Salvador (2007): "Emergencia de lo indígena en la arena política: ¿Un efecto no deseado de la gobernanza?”. En MARTÍ I PUIG, Salvador (ed.), Pueblos Indigenas y politica en América Latina: El reconocimiento de sus derechos y el impacto de sus demandas a inicios del siglo xxi (Barcelona, Fundación CIDOB).

MARTÍ I PUIG, Salvador (2010): “The emergence of Indigenous movements in Latin America and their impact on the Latin American political scene. Interpretive tools at the local and global levels". En Latin American Perspectives, vol. 37, No 6, University of Salamanca, pp. 355-370 
PAJUELO, Ramón (2006): "Participación política indígena en la sierra peruana: Una aproximación desde las dinámicas nacionales y locales". En Anthropologica del Departamento de Ciencias Sociales, vol. 25, No 25 , Pontificia Universidad Católica del Perú, pp. 199-202

RADCLIFFE, Sarah (2007): “Tejiendo redes: Organizaciones y capital social en los pueblos indígenas". En MARTÍ I PUIG, Salvador (ed.) Pueblos indígenas y política en América Latina: El reconocimiento de sus derechos y el impacto de sus demandas a inicios del siglo xxi (Barcelona, Fundación CIDOB).

SCHILLING-VACAFLOR, Almut (2012): "Democratizing Resource Governance through Prior Consultations? Lessons from Bolivia's Hydrocarbon Sector”. En German Institute of Lobal and Area Studies, Working Papers, № 184, pp. 1-27.

SCHMELKES, Sylvia (2009): "Interculturalidad, democracia y formación valoral en México”. En Revista Electrónica de Investigación Educativa, vol. 11, No 2, Universidad Iberoamericana, pp. 1-10.

SPINNER-HAVEL, Jeff (200o): "Land, Culture and Justice: A Framework for Group Rights and Recognition”. En Journal of Politcal Philosophy, vol. 8, No 3 , University of North Carolina.

STAVENHAGEN, Rodolfo (2012): "Pueblos indígenas: Retos después de la batalla”. En GÓMEZ, Felipe (ed.) y BERRAONDO, Mikel (ed.), Los derechos indígenas tras la Declaración (Bilbao, Universidad de Deusto), pp. 15-38.

TOMASELLI, Alexandra (2016) Indigenous Peoples and their Right to Political Participation: International Law Standards and their Application in Latin America (Baden-Baden, Nomos).

WRIGHT, Claire (2014): "Indigenous Mobilisation and the Law of Consultation in Peru. A Boomerang Effect?”. En International Indigenous Policy Journal, vol. 5, № 4, Universidad Autónoma de Nuevo León, pp. 1-16

WRIGHT, Claire y AGUIRRE, Víctor (2015): "Grupos vulnerables en el escenario político". En ARANGO, Xóchitl (coord.) y HERNÁNDEZ, Abraham (coord.), Ciencia Política. Perspectiva Multidisciplinaria (México, D.F, Tirant lo Blanch), pp. 167-180.

ZORNOZA, Juan (2011): Mecanismos de gestión pública ciudadana en una democracia multiétnica y pluricultural. Un estudio de caso. En Forum, Universidad Nacional de Colombia, No 2, pp. 127-153. 